\title{
Gasto en alimentos y bebidas fuera del hogar. El caso de México, 1992 y 2008*
}

\author{
Ignacio Llamas Huitrón, ** Humberto Armando Charles Leija*** \\ y Gilberto Aboites Manrique ****
}

\section{RESUMEN}

Se elaboró un modelo teórico con supuestos de preferencias endógenas al hogar, contextual y socialmente dependientes, a fin de analizar los principales factores determinantes de la probabilidad de que un hogar gastara en alimentos y bebidas fuera del hogar en México, durante 1992 y 2008. Las variables explicativas de dicha probabilidad fueron las relacionadas con el hogar en su conjunto - decil de ingreso-, con el jefe del hogar-sexo, edad y nivel de estudios formales-, con el resto de los miembros del hogar-número, insertos en el mercado laboral, estudiantes becados, menores de 12 años y mayores de 65-, y con la localidad del hogar -rural o urbana-. Se utilizó un modelo logit binomial y los microdatos de la Encuesta Nacional de Ingreso y Gasto de los Hogares 1992 y 2008. Las variables que más influyeron en la probabilidad de consumo fuera del hogar en sentido positivo fueron el decil de ingreso, el nivel de escolaridad del jefe y la localidad urbana, mientras que en sentido negativo fueron el sexo femenino del jefe del hogar y su edad, el tamaño del hogar y el número de menores de 12 años de edad y de mayores de 65 .

Palabras clave: hogar, consumo, gasto alimentario, mercado laboral, logit binomial.

Clasificación JEL: D12, D13, E21, L66.

\begin{abstract}
A model was built in order to deal with the determinants of the probability that a household expends in food and drinks away from home, in Mexico for the years of 1992 and 2008. The variables taken into consideration as determinants were: household's income decile; age, sex, and education level of household's head; household size, household's members in the labor market, number of scholarship receivers, number of household's members under 12 and above 65 years old; and household urban or rural site. A logit model was used to fit the data of Encuesta Nacional de Ingreso y Gasto de los Hogares 1992 and 2008. The variables that more positively impact the probability of consumption away from home were the household income decile, the level of education of household's head, and household urban site while household head's female and age, household size, and the number of members under 12 and above 65 years old had a negative effect in such a probability.
\end{abstract}

Keywords: household, consumption, food expenditure, labour market, binomial logit. JEL classification: D12, D13, E21, L66.

* Fecha de recepción: 07/02/2012. Fecha de aprobación final: 29/08/2012.

** Profesor investigador de la Universidad Autónoma Metropolitana, Unidad Iztapalapa. Correo electrónico: 1lamas.huitron@hotmail.com

*** Centro de Investigaciones Socioeconómicas (CISE) de la Universidad Autónoma de Coahuila. Correo electrónico: humbertocharles@yahoo.com

**** Investigador del Centro de Investigaciones Socioeconómicas (CISE) de la Universidad Autónoma de Coahuila. Correo electrónico: g_aboites@yahoo.com.mx 


\section{INTRODUCCIÓN}

Este artículo analiza las principales variables que determinan la probabilidad de que se realicen gastos en alimentos y bebidas fuera del hogar (GABFH) por medio de un modelo que sistematiza las relaciones entre las variables explicativas y el gasto.

El marco teórico se fundamenta en los enfoques institucionalista y evolucionista, los cuales cuestionan los supuestos básicos de la teoría económica neoclásica sobre un agente (ya sea el individuo o el hogar) omnisciente con preferencias dadas.

Por el contrario, se parte de que el hogar desarrolla sus esquemas de preferencias a partir de experiencias -de prueba y error de las canastas que puede adquirir y de las tecnologías que puede usar para transformarlas en bienes de consumo- y que, por lo tanto, las decisiones se toman con información limitada. En otras palabras, se sostiene que las preferencias para consumir dentro o fuera del hogar dependen del contexto y son endógenas.

Las variables elegidas para explicar la probabilidad de que se realice GABFH fueron la pertenencia del hogar a un decil de ingreso; sexo, edad y nivel de estudios formales del jefe del hogar; número de miembros del hogar, número de miembros que participan en el mercado laboral, número de estudiantes becados, menores de 12 y adultos mayores de 65 años; así como el tipo de localidad (rural o urbana). Para poner a prueba las hipótesis planteadas sobre la determinación de la probabilidad de que ocurra GABFH se utilizó un modelo logit binomial y los microdatos de la Encuesta Nacional de Ingreso y Gasto de los Hogares de 1992 y de 2008.

El texto consta de seis secciones. La primera plantea el marco teórico usado para construir el modelo con el que se sistematizaron las variables elegidas para determinar la probabilidad de que un hogar realice GABFH. En la segunda se exponen las principales hipótesis de trabajo o proposiciones analíticas empíricamente verificables a partir del análisis estadístico realizado.

La tercera presenta la metodología utilizada para el análisis empírico: definición de variables y el logit binomial. En la cuarta se presentan algunos resultados estadísticos de las principales características de las variables utilizadas. La quinta se ocupa de analizar los resultados econométricos tomando como punto de referencia las hipótesis planteadas. En la última sección, se plantean algunas reflexiones finales acerca de los resultados y del tema de estudio. 


\section{EL MODELO TEÓRICO}

La unidad de análisis es el hogar. Su comportamiento es la búsqueda de la maximización del bienestar (presente y futuro) a través del consumo, así como de la inversión en capital físico y humano. El hogar es un elemento dentro de un sistema de hogares y tiene la capacidad de aprender de la interacción con sus pares y con otros sistemas y, por lo tanto, construir y adaptar sus preferencias sociales, así como sus preferencias de bienes y servicios de consumo.

Se adopta el enfoque institucionalista que reconsidera la búsqueda del interés propio como el supuesto fundamental de la teoría económica e introduce los valores sociales de solidaridad y reciprocidad en el bienestar del hogar. Esto no significa que se modifique el papel central que en el enfoque convencional juegan las preferencias, creencias y restricciones para explicar el comportamiento de los hogares, aunque sí implica modificar el modelo convencional en tres aspectos (Bowles, 2004, cap. 3):

1) El comportamiento del hogar se explica mejor por las preferencias sociales: al actuar, el hogar generalmente toma en cuenta las consecuencias de sus acciones tanto para sí como para los demás. A menudo hay preocupación no sólo por las consecuencias sino también por las intenciones de los otros hogares. Un ejemplo importante de las preferencias sociales son los motivos de reciprocidad, los cuales explican que un hogar muestre generosidad con quienes se portan bien (con él o con otros) mientras que castiga a quienes no lo hacen.

2) El hogar tiene un comportamiento adaptativo en la observancia de reglas. Significa que actúa conforme a reglas elementales, simples, en la toma de decisiones de acuerdo con recursos cognitivos limitados. Entre las reglas de comportamiento desarrolladas se encuentran las prescripciones éticas que gobiernan las acciones hacia y con los otros, o sea, las normas sociales con las cuales las acciones son evaluadas por el actor (cuando la norma ha sido internalizada) y respaldadas por la sanción social.

El hogar posee racionalidad limitada dada la información disponible, la capacidad intelectual y el tiempo para tomar decisiones. Además, es común que las decisiones de gasto se tomen bajo condiciones de información incompleta respecto a los bienes y servicios adquiridos y, en algunas ocasiones, motivadas por alguna emoción (French, 1986). Así, el hogar se encuentra en constante proceso de prueba y error a la hora de 
elegir qué y dónde gastar en bienes de consumo. Dicho proceso le permite encontrar los bienes que procuran una mayor satisfacción o bienestar. Es decir, las preferencias no están "dadas", puesto que durante toda su existencia el hogar pasa por un proceso de experimentación. De este modo formula reglas que provienen de la experiencia y, si funcionan, las sigue usando; cuando presentan características insatisfactorias, se cambian (Earl, 1995).

3) El comportamiento depende del contexto. Se utiliza información contextual para determinar el comportamiento apropiado en cualquier situación. Y se evalúa el resultado desde un punto de vista particular, específicamente desde el estado real o desde el estado experimentado por el hogar de referencia. Por eso se afirma que las preferencias son en cierta medida más aprendidas que exógenamente dadas. Los cambios duraderos en el comportamiento de un hogar a menudo resultan de su propia experiencia. Esto significa que los hogares que experimentan diferentes estructuras de interacción social por periodos prolongados probablemente muestren diferentes comportamientos, no simplemente porque las restricciones e incentivos vinculados a estas instituciones son diferentes sino también porque la estructura de la interacción social influye en la evolución de los repertorios de comportamiento de los hogares, en la forma en que las situaciones inducen comportamientos y la forma en la que se evalúan los resultados. Finalmente, las instituciones sociales influyen en cuanto a quién se conoce, para hacer qué y con qué resultados o recompensas. Como resultado, las motivaciones se estructuran a través del proceso cultural o incluso de la transmisión genética. Entonces, las preferencias son contextualmente especificas y endógenas.

Existen diversos elementos que modifican las preferencias del hogar; por ejemplo: su composición, la localidad, la interacción con el medio ambiente y los vecinos, el tiempo disponible para dedicarlo a producir bienes domésticos o convivir con los seres queridos. Esto explica que en determinada situación un hogar decida consumir o no un determinado producto dentro o fuera del mismo Becker (1991) propuso que los integrantes de cada hogar buscan maximizar su utilidad asignando de manera óptima no sólo su ingreso, sino también el tiempo disponible. Afirmó que los hogares más eficientes son aquellos que tienen, cuando menos, un individuo dedicado íntegramente a las actividades domésticas (no remuneradas) y otro al trabajo remunerado. Ve al hogar como una pequeña fábri- 
ca donde se producen bienes y servicios. Un miembro del hogar puede "fabricar" bienes caseros que satisfagan necesidades (comida, hijos, afecto), mediante el uso de insumos (encontrados en el mercado), capital físico, habilidades y tiempo. Entonces, cuando un miembro del hogar cocina un pastel para comer en casa no paga directamente un precio de mercado, sino un precio sombra, igual al costo de producción (ingredientes, gas, uso del horno) más el costo de oportunidad del tiempo dedicado a la elaboración (que pudo haber empleado en alguna actividad remunerada o de esparcimiento).

En resumen: el problema del hogar es la elección de la canasta y de la tecnología para procesarla en bienes de consumo en un contexto de información imperfecta. Al afrontar la complejidad inherente a la elección, el hogar desarrolla sus preferencias esquemáticas. Éstas son reglas que forman las bases de un modelo de interacción del hogar (Potts, 2000). En consecuencia, las decisiones de gasto alimentario del hogar dependen de sus preferencias sobre los bienes y están sujetas a restricciones. Economiza recursos monetarios y cognitivos limitados de acuerdo con reglas desarrolladas a través de la experiencia. Así, en este artículo se analizan tales preferencias y restricciones cuando se elige gastar en alimentos y bebidas fuera del hogar (GABFH). A continuación se presenta el modelo con el que se realiza dicho análisis.

Para identificar y analizar los determinantes de la probabilidad de GABFH en México, se consideraron las variables demográficas del hogar. Se separaron, con fines analíticos, las relacionadas con el jefe y las relacionadas con el resto de los miembros del hogar (gráfica 1). El conjunto de estas variables más la de localidad (urbana o rural) se consideraron determinantes de la probabilidad de GABFH. Por esta razón se afirma que las preferencias del hogar son endógenas, pues dependen de la estructura del hogar y su contexto.

Gráfica 1. Modelo de determinación de la probabilidad de GABFH

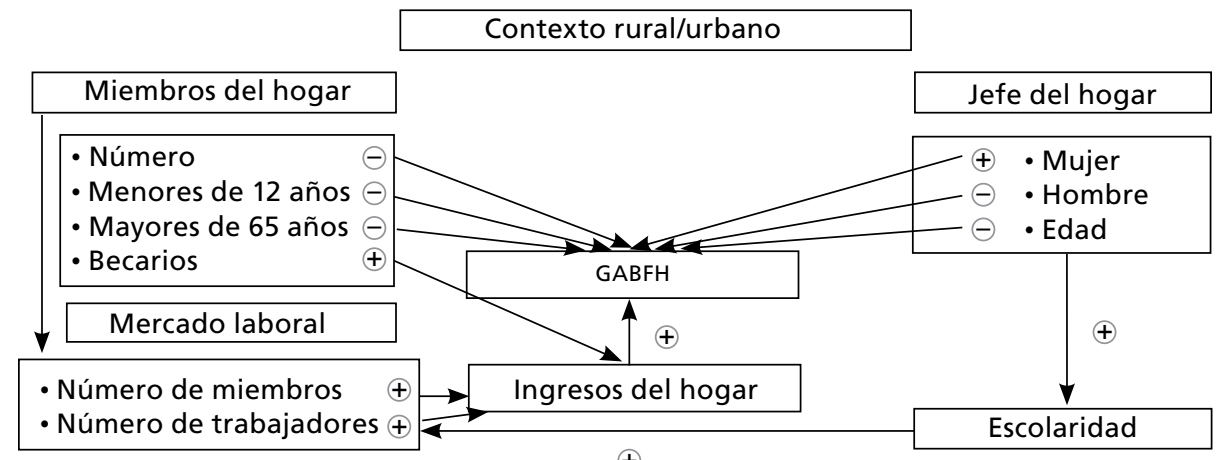




\section{Variables relacionadas con el jefe del hogar}

Se consideró pertinente el sexo. Si el jefe es hombre se esperaría que la probabilidad de GABFH fuera menor que si es mujer. La explicación se encuentra en la tradicional división del trabajo que ha persistido en la sociedad, que considera al varón como el principal proveedor de recursos del hogar y a la mujer como ama de casa encargada de la producción y provisión de satisfactores domésticos. Esta especialización reduce los costos de oportunidad de consumo en el hogar en relación con el consumo fuera, con la consecuente reducción en GABFH. Por el contrario, si el jefe del hogar es mujer se espera que ésta divida su tiempo entre actividades remuneradas fuera del hogar y actividades de producción doméstica. Esta división reduce el tiempo disponible para la producción de bienes en el hogar, lo cual aumenta el costo de oportunidad de producirlos e incrementa, en consecuencia, la propensión al GABFH.

También se consideró como variable pertinente la edad del jefe del hogar. El efecto de ésta en la probabilidad de GABFH es doble. Por un lado, a menor edad, es de esperar que el jefe del hogar disponga de más energía para involucrase en actividades productivas (doble trabajo, horas extras) y sociales (frecuentar amistades, esparcimiento), lo cual tendría un efecto positivo en la ocurrencia de GABFH; sin embargo, también es probable que a temprana edad, el capital humano (físico e intelectual) e institucional (antigüedad en el trabajo, prestaciones diversas) del jefe no genere rendimientos suficientes que le permitan el consumo fuera del hogar, lo cual tendría un efecto negativo para que se dé el GABFH. Por otro lado, a mayor edad, es de esperar menor involucramiento del jefe en las actividades productivas y sociales señaladas, lo cual tendría un efecto igualmente negativo en la ocurrencia de GABFH; sin embargo, es probable que su capital humano e institucional genere rendimientos suficientes que le permitan el consumo fuera del hogar, lo cual tendría un efecto positivo en la probabilidad de GABFH. El efecto neto final en la probabilidad de GABFH es una cuestión de prueba empírica, la cual se realizará en este estudio.

Por su parte, Gourinchas y Parker (1999) identificaron que los jóvenes estadunidenses tienen mayor propensión a gastar en bienes no duraderos, mientras que los adultos se inclinan más al ahorro. Por ello, se esperaría que en un hogar con un jefe menor de 40 años haya más propensión a erogar en comidas fuera de casa.

Finalmente, se consideró el nivel educativo del jefe del hogar. Se espera que un mayor nivel educativo tenga dos efectos positivos en la probabilidad de 
GABFH. Primero, un mayor nivel de escolaridad permite aspirar y acceder a mejores puestos laborales y, con ello, a una mejor remuneración, lo cual aumenta la probabilidad de GABFH. Segundo, la escolaridad cambia los hábitos, costumbres, valores y actitudes de las personas. Por ello, es de esperar que a mayor nivel de escolaridad aumente el gusto por la diversidad y la apreciación culinaria $\mathrm{y}$, con ello, aumente la probabilidad de GABFH.

Existe evidencia de que en Estados Unidos los individuos altamente educados "posponen" el consumo; tienen su mayor nivel de consumo alrededor de los 50 años, mientras que aquellos de baja escolaridad, bachillerato incompleto y menos, lo alcanzan, en promedio, a los 37 (Attanasio, Banks, Meghir y Weber, 1995). Por ello, es probable que a mayor edad y nivel educativo del jefe del hogar aumente el GABFH.

Por otro lado, Attanasio y Székely (1999) señalan que en México los hogares con mayor nivel educativo aportan la mayor parte del ahorro proveniente de las familias (55.7\% del total en 1996). Por ello, se esperaría que los hogares mexicanos de los primeros deciles gasten un mayor porcentaje de sus ingresos en consumo de bienes no duraderos, entre ellos, alimentos y bebidas fuera del hogar.

En relación con el efecto del nivel de escolaridad en el GABFH, Attanasio, Banks, Meghir y Weber (1995) observaron que en los hogares de EU donde el jefe posee educación universitaria, la mujer trabaja menos horas fuera del hogar durante el periodo de crianza de los hijos; es decir, en este tipo de hogar se libera a la mujer de la responsabilidad de aportar ingresos al hogar para que pueda dedicar más tiempo al cuidado de los menores de edad. Este hecho indica que la probabilidad de GABFH también dependerá del número de miembros del hogar, así como de su edad.

\section{Variables relacionadas con otros miembros del hogar}

En esta categoría se incluye el número de miembros del hogar como determinante de la probabilidad de GABFH, que tiene dos efectos encontrados sobre ésta. Primero, a mayor número es de esperar que aumenten las restricciones monetarias, pues los recursos por miembro del hogar se reducen y con ello la probabilidad de GABFH. Pero, segundo, también aumenta la participación potencial de ellos en el mercado laboral y, por ende, la posibilidad de disponer de mayores recursos en el hogar y un aumento en la probabilidad de GABFH.

Asimismo, se consideró el número de menores de 12 años. Una primera reflexión indica que una mayor cantidad de menores en el hogar implica una menor 
propensión a consumir fuera, debida a los cuidados que necesitan y a los gastos involucrados en su manutención, los cuales reducen el ingreso disponible del hogar para otros usos, como el GABFH.

También se consideró el número de adultos mayores de 65 años. Aunque esta variable tiene efectos opuestos en la probabilidad de GABFH, se espera que una mayor presencia de adultos mayores de 65 años la inhiba. Lo anterior, debido a dos razones: primera, que los adultos mayores generalmente necesitan atención especial, la cual requiere recursos que el hogar substrae de otros usos posibles, incluido el gasto en consumo fuera del hogar. Segunda, una parte importante de los adultos mayores son productivos fuera y dentro del hogar, y cuando permanecen en éste pueden colaborar en la producción de bienes domésticos, lo cual afecta negativamente el GABFH. El efecto opuesto se presenta cuando los adultos mayores reciben pensión o jubilación; en este caso, contribuyen al ingreso del hogar y con ello aumentan la probabilidad de GABFH.

Como curiosidad analítica, se incluyó en esta categoría el número de miembros perceptores de transferencias. Un estudio realizado en EU (Kinsey, 1983) sobre esposas trabajadoras y consumo fuera del hogar muestra que los ingresos generados por los jóvenes trabajadores de tiempo parcial afectan positivamente el GABFH. En esta investigación se pone a prueba si los ingresos de becarios afectan de manera estadísticamente significativa el consumo fuera del hogar ya que las becas constituyen (al igual que las percepciones de los trabajadores de tiempo parcial) un aumento en el ingreso disponible por el hogar para distintas opciones de uso, incluido el GABFH.

\section{Variable institucional: el mercado de trabajo}

Este mercado es la institución en la que el hogar ofrece sus servicios productivos a cambio de ingresos. Qué tantos ingresos obtenga el hogar dependerá del número de miembros que trabajen, del ingreso por hora trabajada y del número total de horas trabajadas. Éstas son tres variables que usa el hogar es su estrategia de supervivencia, con la cual busca obtener el máximo de bienestar dados los recursos a su disposición. A mayor número de miembros en el mercado laboral mayor es el ingreso esperado del hogar y mayor la probabilidad de GABFH.

También a mayor cantidad de horas trabajadas por los miembros del hogar, mayor ingreso y mayor probabilidad de GABFH. El ingreso por hora trabajada depende de una estrategia de largo plazo del hogar, la cual implica básicamente la acumulación de capital humano (escolaridad y capacitación) de 
sus miembros a edades tempranas y no fue considerada como una variable institucional en este estudio.

\section{Variable de contexto: localidad urbana o rural}

Las preferencias del hogar están estructuradas y parcialmente determinadas por el contexto: la convivencia con los pares (preferencias sociales en el vecindario, en el lugar de trabajo, en la iglesia) y la localidad de residencia (urbana o rural). En consecuencia, la probabilidad de GABFH será mayor en las áreas urbanas que en las rurales, lo que se explica porque las distancias que los miembros del hogar tienen que recorrer para llegar al lugar de destino, sea de estudio, trabajo o mera convivencia, consumen más tiempo en las áreas urbanas que en las rurales, lo cual propicia el GABFH. Además, las áreas urbanas tienen mayor infraestructura de oferta de servicios de consumo, lo que incentiva el GABFH.

En resumen, en el modelo propuesto se supone que la estrategia de supervivencia del hogar, con el máximo de bienestar posible se realiza tomando en cuenta: 1) sus restricciones de ingreso (número de miembros del hogar perceptores de ingreso y monto percibido), 2) sus preferencias endógena y socialmente determinadas por sexo, educación y edad del jefe del hogar; el tamaño del hogar, la presencia de menores de edad y de adultos mayores, así como por el contexto (tipo de localidad que habitan y relaciones sociales).

\section{HIPÓTESIS DE TRABAJO}

Se llama hipótesis de trabajo a las proposiciones analíticas empíricamente verificables que se presentan a continuación. Los efectos esperados de las variables en la probabilidad de GABFH son los efectos netos, ya que, como se planteó en la sección anterior, algunas variables tienen efectos opuestos.

H1. A mayor decil de ingreso se espera una mayor probabilidad de GABFH. Se considera que los bienes consumidos fuera del hogar son bienes normales. ${ }^{1}$ Por lo tanto, a mayor nivel de ingreso del hogar, mayor GABFH. Además, es probable que un incremento en los ingresos del hogar se deba al aumento del tiempo que

\footnotetext{
${ }^{1}$ Un bien normal es aquel que el hogar consume más a medida que recibe más ingresos. Si el consumo del bien aumenta proporcionalmente más que el aumento en sus ingresos, entonces se define como un bien de lujo (Varian, 1992, p. 117). Es probable que el GABFH sea un bien de lujo para los hogares de mayores ingresos; este tema es materia de otra investigación.
} 
se dedica a actividades laborales remuneradas, lo que a su vez disminuye el lapso disponible para preparar alimentos en el hogar y eleva la propensión a consumir fuera del hogar. Es probable también que los hogares en una mejor situación económica valoren más su tiempo libre y lo ocupen en socializar y convivir con la familia aumentando su GABFH.

H2. Se espera que la probabilidad de GABFH sea mayor cuando el jefe del hogar es mujer que cuando es hombre. Lo anterior se supone como resultado de la probable asignación de parte de su tiempo a actividades remuneradas fuera del hogar y la consecuente disminución del dedicado a quehaceres del hogar.

H3. A mayor edad del jefe del hogar se espera una menor probabilidad de GABFH. A mayor edad del jefe se espera un menor involucramiento en las actividades productivas y sociales señaladas en el apartado I.1, lo cual tendría un efecto negativo en la probabilidad de GABFH.

H4. A mayor nivel de escolaridad del jefe del hogar se espera una mayor probabilidad de GABFH. Generalmente, las personas con mayor nivel de escolaridad tienen mayor nivel de ingresos y más acceso a información sobre alternativas de consumo de alimentos y bebidas.

H5. A mayor número de miembros del hogar se espera una menor probabilidad de GABFH. En consecuencia, el hogar unipersonal tendría mayor probabilidad de GABFH que el resto, pues las personas que viven solas dividen su tiempo entre trabajo remunerado y labores domésticas, con lo cual aumenta el costo de oportunidad de las actividades domésticas y aumenta la propensión a consumir fuera del hogar. En cambio, los hogares con varios miembros pueden desarrollar una eficaz división del trabajo entre actividades remuneradas y domésticas, con lo cual se esperaría una menor probabilidad de GABFH.

H6. A mayor número de dependientes en el hogar se espera una menor probabilidad de GABFH. La presencia de menores de 12 años y mayores de 65 inhibe el GABFH. Los menores demandan atención y los adultos mayores, por lo general, disponen de más tiempo libre que pueden destinar a las labores domésticas; esto último aumenta el costo relativo de consumir fuera del hogar (Prochaska y Schrimper, 1973) con el consecuente efecto negativo sobre la propensión al GABFH. 
H7. A mayor número de jóvenes perceptores de transferencias en el hogar se espera una mayor probabilidad de GABFH. Las transferencias constituyen una renta pura para los hogares, lo que suaviza las restricciones monetarias para realizar mayor gasto en general, incluido el GABFH.

H8. A mayor número de miembros en el mercado laboral aumenta la probabilidad de GABFH. Esta circunstancia aumenta los ingresos esperados del hogar así como el movimiento de sus miembros que laboran a su lugar de trabajo y con ello la probabilidad de GABFH.

H9. A mayor número de horas semanales trabajadas por los miembros del hogar, mayor probabilidad de GABFH. Mientras mayor es el número de horas trabajadas, más tiempo pasan fuera del hogar sus miembros ocupados y con ello ocurre mayor probabilidad de GABFH.

H10. Se espera que el hogar urbano tenga una probabilidad de GABFH mayor que el hogar rural. El tamaño de la localidad es una variable de contexto que influye positivamente en la probabilidad de GABFH. Este gasto es un fenómeno fundamentalmente urbano; los habitantes de las ciudades pasan más tiempo en traslados que los del campo, lo que inhibe el consumo en casa. Asimismo, las ciudades tienen una mayor disponibilidad de establecimientos de bebidas y alimentos preparados.

\section{METODOLOGÍA DEL ANÁLISIS EMPÍRICO}

Para poner a prueba las hipótesis planteadas se utilizó un modelo logit binomial. El resultado de este modelo se interpreta como la probabilidad de que una observación (hogar) pertenezca al grupo codificado con la unidad. Se identifican las variables más importantes que impactan en la probabilidad de que un evento ocurra; en este caso, que el hogar tenga GABFH. Además, el modelo permite jerarquizar las variables explicativas según su influencia en la probabilidad de GABFH.

En lenguaje matricial, el modelo logit binomial tiene la especificación general siguiente:

$$
\operatorname{Ln}\left(\frac{Y}{1-Y}\right)=\sum \beta X+u
$$


donde $Y$ es un vector de variables binarias con valor de 1 si el hogar gasta en alimentos y bebidas fuera y 0 si esto no sucede. $X$ es el conjunto de vectores de variables explicativas: decil de ingreso; sexo, edad y educación formal del jefe; tamaño del hogar, cantidad de ocupados, menores de 12 años, mayores de 65, becarios; y localidad rural o urbana. El vector $\beta$ de los coeficientes de regresión son las incógnitas del modelo y representan la influencia de las variables explicativas en la variable dependiente; $u$ representa los residuos no explicados por el modelo (Greene, 2000; Lara y Llamas, 2010). Se utilizaron los microdatos reportados por la Encuesta Nacional de Ingreso y Gasto de los Hogares (ENIGH) del Instituto Nacional de Estadística y Geografía (INEGI) en los años 1992 y 2008. A continuación, se enumeran las variables involucradas en la estimación del modelo.

La variable dependiente:

$\mathrm{GABFH}=0$ si el gasto trimestral en alimentos y bebidas fuera del hogar fue una proporción menor al $10 \%$ del gasto total en bebidas y alimentos; $=1$ si dicha proporción fue mayor o igual a $10 \%$.

Las variables independientes explicativas se especificaron de la manera siguiente:

Decil $=$ dummies de los deciles de ingreso trimestral del hogar (grupo base: decil I).

Sexo $=1$ si el jefe de hogar es mujer (grupo base: el jefe del hogar es hombre).

Edad $=$ años del jefe del hogar.

Ed_formal = dummies del nivel de escolaridad del jefe del hogar; se especificaron cuatro niveles: primaria completa e incompleta; secundaria completa e incompleta; educación media superior completa e incompleta, y educación superior completa e incompleta (grupo base: primaria). 
Tam_hog = número de miembros del hogar. ${ }^{2}$

Menores $=$ número de miembros del hogar menores de 12 años.

P65mas $=$ número de miembros del hogar mayores de 65 años.

Becarios $=1$ si algún integrante del hogar recibe beca; $=0$ en caso contrario. Esta información está disponible sólo para 2008.

N_ocup $=$ número de miembros del hogar, mayores de 14 años, en el mercado laboral.

Hrs_semana $=$ número de horas trabajadas semanalmente por los miembros del hogar.

Rururb $=1$ si el tamaño de localidad es $\geq 15000$ habitantes (urbana); $=0$ si la localidad es $<15000$ habitantes (rural).

En la realización del trabajo econométrico se utilizó el paquete estadístico SPSS.

\section{CARACTERÍSTICAS PRINCIPALES DE LAS VARIABLES UTILIZADAS}

En esta sección se presentan algunas características de los hogares relacionadas con el GABFH en los años 1992 y 2008.

En el cuadro 1, se observa que entre los dos años de estudio se presentó un aumento en el porcentaje de hogares con jefatura femenina. Este hecho está asociado con la disminución observada en el promedio de miembros del hogar, pues la mayoría de las jefas de hogar no viven con un cónyuge (Aboites y Félix, 2010), fenómeno que ha influido en la reducción de la tasa de natalidad. En consecuencia, como se puede observar, el promedio de menores de 12 años en el hogar ha disminuido en los últimos años. También se puede observar que el porcentaje de hogares con jefatura femenina declina a medida que se asciende en la escala de los deciles de ingreso.

\footnotetext{
${ }^{2}$ No incluye trabajadores domésticos, huéspedes ni personas de parentesco no especificado.
} 
Cuadro 1. Características de las variables del modelo logit binomial. México, 1992 y 2008

\begin{tabular}{|c|c|c|}
\hline Variables & 1992 & 2008 \\
\hline \multicolumn{3}{|l|}{ GABFH/GAT* por decil de ingreso (porcentaje del GAT) } \\
\hline Decil । & 2.9 & 5.3 \\
\hline Decil ॥ & 6.3 & 8 \\
\hline Decil III & 6.5 & 9.3 \\
\hline Decil IV & 7.7 & 10.6 \\
\hline Decil v & 7.8 & 11.6 \\
\hline Decil vı & 9.3 & 13.2 \\
\hline Decil VII & 8.3 & 14.8 \\
\hline Decil VIII & 13.7 & 17.7 \\
\hline Decil IX & 12.7 & 20.7 \\
\hline Decil $x$ & 18.5 & 27.2 \\
\hline GABFH/GAT promedio (porcentaje del GAT) & 9.4 & 13.8 \\
\hline Jefe mujer (porcentaje de hogares) & 14.2 & 24.7 \\
\hline Decil I & 17.6 & 30 \\
\hline Decil $x$ & 11.9 & 17.1 \\
\hline Educación del jefe (nivel promedio) & Secundaria incompleta & Secundaria incompleta \\
\hline \multicolumn{3}{|l|}{$\begin{array}{l}\text { Distribución del nivel educativo del jefe (porcentaje } \\
\text { de hogares) }\end{array}$} \\
\hline Primaria & 66 & 50 \\
\hline Secundaria & 16 & 25 \\
\hline Bachillerato & 8 & 11 \\
\hline Superior & 11 & 14 \\
\hline Edad del jefe (promedio de años) & 42.9 & 48.2 \\
\hline Miembros del hogar (promedio de personas) & 4.7 & 4 \\
\hline Menores de 12 años (promedio de personas) & 1.4 & 0.9 \\
\hline Adultos mayores de 65 años (promedio de personas) & 0.2 & 0.3 \\
\hline $\begin{array}{l}\text { Miembros en el mercado laboral (promedio de } \\
\text { personas) }\end{array}$ & 1.6 & 1.7 \\
\hline $\begin{array}{l}\text { Horas trabajadas por semana por hogar (promedio } \\
\text { de horas) }\end{array}$ & 38 & 45.4 \\
\hline Hogares urbanos (porcentaje de hogares) & 62.4 & 66.2 \\
\hline
\end{tabular}

Fuente: Elaboración propia con base en microdatos de la ENIGH 1992 y 2008.

* Gasto en alimentos y bebidas total, dentro y fuera del hogar.

** Incluye niveles terminados e incompletos. 
El nivel de educación formal del jefe del hogar se mantuvo en secundaria incompleta -nivel que incluye entre más de 6 y menos de 9 años de escolaridad- en los años analizados. Este hecho es coherente con el aumento observado en la escolaridad de la población mexicana de 15 o más años, la cual pasó de 7.0 años en el ciclo escolar 1995-1996 a 8.5 en el ciclo 2008-2009 (INEGI, 2010, cuadro 4.8).

Como se puede observar, el aumento fue insuficiente para superar el nivel de secundaria incompleta. Una consecuencia de ese aumento en años de escolaridad fue que el porcentaje de jefes con primaria (completa e incompleta) disminuyó en $16 \%$, caída que se reflejó en el aumento porcentual del resto de los niveles educativos.

En cuanto al promedio de años de edad del jefe, se identificó un aumento. En 2008, los jefes de hogar eran, en promedio, 6 años mayores que en 1992, lo cual refleja el envejecimiento de la población mexicana. Asimismo, se observó un leve incremento en el promedio de adultos mayores presentes en el hogar. Estos dos elementos, el aumento en la edad del jefe y en la presencia de adultos mayores de 65 años, son factores que probablemente inhiben la propensión a consumir alimentos y bebidas fuera del hogar.

En los años analizados, aumentó el promedio de miembros del hogar en el mercado laboral a pesar de la disminución del tamaño medio de los hogares. Este hecho se origina en la crítica situación económica de las familias, la cual obliga a integrar un mayor número de miembros al mercado laboral para satisfacer las necesidades de ingresos y bienestar. Lo anterior ocasionó un incremento considerable en el promedio de horas a la semana trabajadas por los miembros económicamente activos del hogar. Estos dos factores afectan positivamente la propensión al GABFH, debido a la mayor generación de ingresos laborales y, a la vez, una menor disponibilidad de tiempo para realizar trabajo en el hogar.

Otro factor que afectó positivamente el GABFH fue el aumento en el porcentaje de hogares urbanos, tendencia que se mantendrá en un futuro previsible.

El GABFH como porcentaje del gasto total en alimentos y bebidas de los hogares (GAT) aumentó en los años estudiados. Así, mientras que en 1992, de cada peso empleado en alimentos y bebidas por los hogares nueve centavos se gastaban fuera, en 2008 esa cantidad aumentó a catorce centavos. Este incremento del GABFH se observa en todos los deciles de ingreso. Y como era de esperar, la proporción aumenta con el decil de ingreso. Previsiblemente, estas tendencias se mantendrán toda vez que los factores que afectan positivamente al GABFH seguirán creciendo. 
Otros resultados encontrados y no reportados en el cuadro 1 son:

1) En el año 2008, la mayor proporción (40\%) de GABFH se destinó a la comida del mediodía. Este resultado probablemente se deba a lo difícil de regresar a comer en casa a esa hora para quienes laboran o estudian. Un resultado similar obtuvo Fabiosa (2008).

El desayuno resultó la comida con segundo mayor GABFH (29\%). Probablemente se deba a que las familias no disponen de tiempo suficiente en las mañanas para tomar alimentos antes de salir a realizar las actividades cotidianas y eligen la opción de comer fuera, en algún establecimiento cercano al lugar de trabajo o de estudio.

En último lugar, la cena (con 11\%) y otros eventos (con 20\%) fueron las ocasiones que registraron un menor GABFH. Los resultados anteriores sugieren que comer fuera de casa en horas de la noche se relaciona, en una mayor medida, con propósitos de convivencia y socialización que con uno de ahorro de tiempo.

2) El hogar mexicano consume fuera de casa por razones asociadas fundamentalmente con el ahorro de tiempo. Así, la mayor proporción (46.6\%) de transacciones las realiza en loncherías, fondas, taquerías, cocinas económicas y cenadurías, en establecimientos cercanos al centro de trabajo o de estudio. También frecuenta (16.4\% de las transacciones) a vendedores ambulantes. Por ello, se infiere que el GABFH generalmente se presenta en lugares de bajo costo y de consumo rápido.

3) Aun los hogares de los deciles más altos (IX y X) tienen como lugar más frecuentado las loncherías, fondas y taquerías, ya que el 39.4\% de su GABFH se realiza en este tipo establecimientos, porcentaje similar al del resto de los deciles. Los primeros deciles (I al VIII) tienen al puesto ambulante como el segundo lugar más frecuentado mientras que los deciles más altos (IX y X) tienen al restaurante.

4) El hogar nuclear (familia directa compuesta por padre, madre e hijo(s)) es el más común en México y también el que realiza más transacciones de GABFH. De acuerdo con Becker (1991), se esperaría que este lugar lo ocupara el hogar unipersonal, ya que dispone de menor tiempo para realizar actividades domésticas, pero no ocurre así. Es muy probable que ello se deba a que la mayoría de los hogares unipersonales se encuentran en los primeros deciles de ingreso y no disponen de recursos que les permitan realizar GABFH con mayor frecuencia. 


\section{RESULTADOS ECONOMÉTRICOS}

Las estimaciones se presentan en el cuadro 2.

Cuadro 2. Variables explicativas de la probabilidad de ocurrencia de GABFH. Coeficientes de regresión logit y jerarquía. México, 1992 y 2008

\begin{tabular}{|c|c|c|c|c|c|c|}
\hline \multirow{2}{*}{ Variables explicativas } & \multicolumn{3}{|c|}{1992} & \multicolumn{3}{|c|}{2008} \\
\hline & $B *$ & $\operatorname{Exp}(B)$ & Jerarquía & $B *$ & $\operatorname{Exp}(B)$ & Jerarquía \\
\hline \multicolumn{7}{|l|}{ Decil de ingreso (base: decil ı): } \\
\hline ॥ & 1.07 & 2.9 & 9 & 0.47 & 1.6 & 10 \\
\hline III & 1.17 & 3.22 & 8 & 0.57 & 1.76 & 8 \\
\hline IV & 1.55 & 4.73 & 6 & 0.73 & 2.07 & 7 \\
\hline $\mathrm{v}$ & 1.54 & 4.65 & 7 & 0.89 & 2.43 & 6 \\
\hline VI & 1.69 & 5.4 & 5 & 1.07 & 2.91 & 5 \\
\hline VII & 1.78 & 5.92 & 4 & 1.2 & 3.31 & 4 \\
\hline VIII & 1.93 & 6.89 & 3 & 1.43 & 4.18 & 3 \\
\hline IX & 2.09 & 8.12 & 2 & 1.6 & 4.97 & 2 \\
\hline$x$ & 2.48 & 11.95 & 1 & 1.92 & 6.8 & 1 \\
\hline \multicolumn{7}{|l|}{ Jefe del hogar } \\
\hline Mujer & 1.23 & 1.13 & 16 & -0.01 & 0.99 & 17 \\
\hline Edad & -0.01 & 0.99 & 17 & -0.02 & 0.98 & 18 \\
\hline \multicolumn{7}{|l|}{ Escolaridad (base: primaria) } \\
\hline Secundaria & 0.06 & 1.21 & 12 & 0.13 & 1.13 & 14 \\
\hline Bachillerato & 0.11 & 1.12 & 14 & 0.38 & 1.46 & 11 \\
\hline Superior & 0.23 & 1.65 & 10 & 0.55 & 1.73 & 9 \\
\hline \multicolumn{7}{|l|}{ Variables del hogar } \\
\hline Tamaño del hogar & -0.14 & 0.87 & 18 & -0.24 & 0.79 & 20 \\
\hline Menores de 12 años & 0.02 & 1.02 & 15 & 0.06 & 1.06 & 15 \\
\hline Mayores de 65 años & -0.17 & 0.85 & 19 & -0.08 & 0.92 & 19 \\
\hline Becarios & & & & 0.19 & 1.21 & 12 \\
\hline \multicolumn{7}{|l|}{ Mercado laboral } \\
\hline $\begin{array}{l}\text { Miembros del hogar en el } \\
\text { mercado laboral }\end{array}$ & 0.22 & 1.24 & 11 & 0.12 & 1.13 & 14 \\
\hline Horas trabajadas por semana & 0.01 & 1.01 & 16 & 0 & 1 & 16 \\
\hline \multicolumn{7}{|l|}{ Contexto } \\
\hline Urbano (base: rural) & 0.13 & 1.14 & 13 & 0.15 & 1.17 & 13 \\
\hline Constante & -2.64 & 0.07 & & -0.49 & 0.61 & \\
\hline $\mathbf{R}^{2}$ de Nagelkerke ${ }^{\star \star}$ & & 0.131 & & & 0.176 & \\
\hline
\end{tabular}

*Todos los coeficientes resultaron significativos para cualquier nivel de significación estadística.

**Toma valores entre 0 y 1 . Estima la relación entre las variables explicativas y la variable logit.

Fuente: Elaboración propia con base en los microdatos de la ENIGH 1992 y 2008. 
Los resultados de 1992 y 2008 muestran que la pertenencia a los nueve deciles de ingreso más altos - II al X-tiene un mayor efecto en la probabilidad de GABFH respecto al primer decil y dicho efecto es creciente a medida que se asciende en la escala. Además, el exponencial beta indica que la jerarquía aumenta a medida que se asciende en la escala de los deciles de ingreso y que, además, es muy superior al del resto de las variables explicativas. Estos resultados apoyan la hipótesis $\mathrm{H} 1$ de que los bienes consumidos fuera del hogar son normales y que los hogares en una mejor situación económica valoran más su tiempo libre y lo ocupan en socializar y convivir con la familia, aumentando su GABFH.

El ser jefe del hogar mujer tuvo efectos opuestos en los dos años de estudio. Por un lado, conforme a lo esperado (H2), en 1992, cuando el jefe del hogar era mujer, la probabilidad de GABFH fue mayor que cuando era hombre. Este resultado apoya la idea de que tener una ocupación remunerada fuera del hogar ocasiona que la mujer disponga de menos tiempo para realizar quehaceres del hogar y, por lo tanto, que se recurra al consumo fuera de casa. Pero, por otro lado, en 2008, un hogar con jefe mujer tuvo menor probabilidad de GABFH que con jefatura masculina. Lo anterior respalda el concepto de que existe una doble jornada de la mujer en las sociedades actuales, pues además de contribuir al sostenimiento del hogar a través de la generación de ingreso en el mercado laboral, debe llegar al hogar a realizar las tareas domésticas; tal vez este proceso de doble jornada haya sido propiciado, al menos parcialmente, por la crisis económica que se manifestó a partir de 2008. Sin embargo, entre las variables explicativas consideradas, la de jefatura femenina del hogar se encuentra en la parte baja de la escala de jerarquía: en el año 1992 ocupó el lugar 16 de 19 variables consideradas y en 2008 el lugar 17 de 20.

Conforme a lo esperado (H3), la edad del jefe está inversamente relacionada con la probabilidad de consumo fuera del hogar. Como se puede observar en los resultados estadísticos, el efecto de un año adicional de edad sobre la probabilidad es bajo. Además, entre las variables explicativas de la probabilidad de GABFH, la edad del jefe se encuentra en la parte baja de la jerarquía: en el lugar 17 de 19 en 1992 y en el lugar 18 de 20 en 2008. El resultado apoya la idea de que a mayor edad del jefe se tiene menor involucramiento en las actividades productivas y sociales, lo cual afecta negativamente la probabilidad de GABFH.

Como era de esperar (H4), el nivel de escolaridad del jefe de hogar está positivamente asociado con la probabilidad de GABFH. Cabe destacar que los niveles de escolaridad se ubican solamente por debajo de los deciles de ingreso en la escala jerárquica de las variables explicativas de la probabilidad de GABFH, 
y que la jerarquía crece a medida que se asciende en la escala de los niveles educativos. Este resultado es congruente con la afirmación de que las personas con mayor nivel de escolaridad tienen mayor nivel de ingreso y acceso a información sobre alternativas de consumo de alimentos y bebidas y, con ello, una mayor probabilidad de GABFH.

Los resultados muestran que a mayor número de miembros del hogar (H5) es menor la probabilidad de GABFH. Esta variable se encuentra en la parte baja de la escala de jerarquía de las variables, pues en 1992 ocupa el lugar 18 de 19, y en 2008 el último lugar, el 20. Estos resultados apoyan la conjetura de que a mayor número de personas crece la posibilidad de división del trabajo, lo cual reduce el costo de oportunidad de los bienes y servicios producidos domésticamente y, con ello, se reduce la propensión a consumir fuera del hogar.

También de acuerdo a lo esperado (H6), los resultados confirman que la presencia de menores de 12 años y mayores de 65 inhibió el GABFH en 1992; sin embargo, en 2008 los resultados estadísticos mostraron que la relación entre número de menores de 12 años y probabilidad de GABFH se invierte mientras que se mantiene la relación negativa entre número de mayores de 65 años y la probabilidad de GABFH.

Estas variables se encuentran en la parte baja de la jerarquía y se puede concluir que existe una relación ambigua entre cantidad de menores de 12 años en el hogar y su efecto en la probabilidad de consumo fuera del hogar y que, tal como se expresó en H6, por lo general, los adultos mayores disponen de más tiempo libre que pueden destinar a la realización de labores domésticas disminuyendo la propensión a consumir fuera del hogar.

Según lo previsto (H7), un mayor número de jóvenes perceptores de transferencias (becas) se traduce en mayor probabilidad de GABFH. En la escala jerárquica, esta variable se encuentra en una posición media, ya que ocupó el lugar 12 de 20 en el año 2008. Las transferencias que recibe el hogar aumentan su nivel de ingresos y, con ello, el consumo de bienes normales, incluido el GABFH.

Los resultados muestran que conforme a lo esperado (H8), mientras más miembros en el mercado laboral tenga el hogar y, mientras más horas trabajadas (H9) acumulen, mayor será la probabilidad de GABFH. Pero en la jerarquía de las variables, el número de miembros en el mercado laboral ocupa una posición media y la cantidad de horas trabajadas una posición baja. Lo anterior refleja que estas dos variables no sólo ocasionan aumento en los ingresos esperados del hogar sino que también aumentan el movimiento de sus miembros ocupados hacia el lugar de trabajo y, con ello, la probabilidad de GABFH. 
Por último, el hogar urbano tiene una mayor probabilidad de GABFH que el rural (H10), en consonancia con lo previsto. Asimismo, el tamaño de la localidad ocupa una posición media en la escala de jerarquía de las variables explicativas. Estos resultados reflejan que los habitantes de las ciudades dedican más tiempo a traslados que los rurales y que tienen mayor disponibilidad de establecimientos de venta de bebidas y alimentos preparados, hechos que estimulan la propensión a consumir fuera del hogar.

\section{RESUMEN Y REFLEXIONES FINALES}

Se argumentó que las preferencias de consumo fuera del hogar se forman a partir de la estructura interna de éste, de sus miembros y de la relación entre ellos. Al respecto, se estableció que las preferencias son endógenas. Además se concibió al hogar como elemento que interactúa con otros hogares y que, en conjunto, forman parte de un sistema de hogares. En esa interacción con sus pares, el sistema hogar influye en las preferencias de los otros, así como es influido en las suyas. Es decir, se concibe al hogar como un sistema de comportamiento adaptativo. En este sentido, se estableció que las preferencias están socialmente determinadas. Las preferencias también están parcialmente determinadas por el contexto social y espacial en el que desarrollan sus actividades de consumo.

Entre las variables explicativas del consumo fuera del hogar utilizadas en el trabajo econométrico de esta investigación, la variable decil de ingreso es la de mayor jerarquía en la determinación de la probabilidad de consumo de alimentos y bebidas fuera del hogar. Este hecho permite afirmar que la canasta de dichos bienes constituye un bien normal. El resto de las variables se pueden considerar de jerarquía media -como el nivel de escolaridad del jefe del hogar (secundaria, bachillerato y superior) y el contexto urbano- o baja -como el sexo, edad del jefe y tamaño del hogar; número de menores de 12 años, de mayores de 65 y de becarios en el hogar; cantidad de miembros en el mercado laboral y de horas trabajadas.

Algunas de las variables explicativas consideradas tienen un efecto positivo y otras un efecto negativo en la probabilidad de consumo fuera del hogar. Entre las primeras se cuentan el decil de ingreso y el contexto urbano, las cuales tienen una tendencia histórica de crecimiento; entre las segundas se hallan el tamaño del hogar, que declina con el tiempo, y el número de adultos mayores de 65 años, el cual va en aumento. Esperamos como resultado neto de este conjunto 
de efectos que el gasto en alimentos y bebidas fuera del hogar (GABFH) continúe creciendo en un futuro predecible.

Ahora bien, la probabilidad de GABFH depende del ingreso y estructura del hogar (sexo, edad y escolaridad del jefe; número de miembros y su participación en el mercado laboral; cantidad de estudiantes becados, de menores de 12 años y de mayores de 65), así como del contexto (tamaño de la localidad). Los actores y el escenario están delimitados y es factible plantear propuestas de política social. Específicamente, se debería facilitar la creación de fuentes de comida rápida -fondas, taquerías y loncherías- para abaratar las opciones de consumo fuera de la casa, como una estrategia de defensa del bienestar de los hogares situados en los deciles de ingresos más bajos, los cuales tienen un porcentaje significativo y creciente de GABFH en establecimientos de comida rápida durante el desayuno y la comida. Sería una estrategia de política tendiente a mantener bajos los precios de esos bienes como defensa del salario real de los trabajadores mediante un mejor acceso y calidad de los alimentos.

La política que se propone requiere instrumentar una campaña educativa de higiene en la preparación y distribución de alimentos y bebidas fuera del hogar. Por ejemplo, se podría impartir instrucción para lavar higiénicamente las verduras (lechuga, jitomate $u$ otras) a fin de que queden libres de bacterias o para mejorar nutricionalmente los alimentos tradicionales.

\section{REFERENCIAS BIBLIOGRÁFICAS}

Aboites, Gilberto, y Félix, Gustavo (2010), Patrones de consumo alimentario en México. Retos y realidades, México, Editorial Trillas.

Attanasio, Orazio P.; Banks, James; Meghir, Costas, y Weber, Guglielmo (1995), "Humps and Bumps in Lifetime Consumption", National Bureau of Economic Research Working Paper No. 5350.

— Hurst, Erik, y Pistaferri, Luigi (2012), "The Evolution of Income, Consumption, and Leisure Inequality in the US, 1980-2010", National Bureau of Economic Research Working Paper No. 17982.

—, y Székely, Miguel (1999), "Ahorro de los hogares y distribución del ingreso en México", Economia Mexicana, Vol. 8, No. 2, pp. 267-338.

Becker, Gary (1991), A Treatise on the Family, Cambrige, MA, Harvard University Press.

Bowles, Samuel (2004), Microeconomics: Behavior, Institutions, and Evolution, New York, Princeton University Press. 
Earl, Peter E. (1995), Microeconomics for Business and Marketing. Lectures, Cases and Worked Essays, Cambridge, UK, Edward Elgar.

Fabiosa, Jacinto F. (2008), "The Food-Away-from-Home Consumption Expenditure Pattern in Egypt", Working Paper 08-WP 474, Iowa State University/Center for Agricultura and Rural Development.

French, Simon (1986), Decision Theory. An Introduction to the Mathematics of Rationality, New Jersey, Prentice Hall.

Gourinchas, Pierre-Oliver, Parker, Jonathan A. (1999), "Consumption Over the Life Cycle”, National Bureau of Economic Research Working Paper No. 7271.

Greene, William H. (2000), Econometric Analysis, cuarta edición, Upper Saddle River, N J, Prentice Hall.

Instituto Nacional de Estadística y Geografía (2010), Estadístico de los Estados Unidos Mexicanos, México, INEGI.

Kinsey, Jean (1983), "Working Wives and the Marginal Propensity to Consume Food away from Home", American Journal of Agricultural Economics, 65(1), pp.10-19.

Lara, Manuel, y Llamas, Ignacio (2010), "Permanencia educativa en los hogares rurales. El caso de México 2005”, en Llamas, Ignacio; Garro, Nora, y Campos, Guillermo (coords.), Politica social: enfoques y análisis, México, UAM-Iztapalapa.

Potts, Jason (2000), The New Evolutionary Microeconomics. Complexity, Competence and Adaptive Behavior, Cheltenham, UK, Edward Elgar.

Prochaska, Fred J., y Schrimper, Ronald A. (1973), “Opportunity Cost of Time and Other Socioeconomic Effects on Away from Home Food Consumption", American Journal of Agricultural Economics. 55, Nov., pp. 595-603.

Varian, Hal R. (1992), Microeconomic Analysis, tercera edición, New York, W.W. Norton \& Co. 